\title{
MODELOS ESTATÍSTICOS E SUA APLICAÇÃO NA ESTIMATIVA DO COEFICIENTE DE PMD MÁXIMO EM ENLACES ÓTICOS
}

\author{
C. E. P. Blume, A. A. P. Pohl e J. A. Medeiros Neto
}

\begin{abstract}
Resumo - Apresentamos neste trabalho um estudo comparativo entre modelos estatísticos utilizados no tratamento do fenômeno de dispersão do modo de polarização em enlaces óticos. Com os resultados obtidos é possível estimar o coeficiente de PMD máximo admissível em sistema de comunicação ótica de longa distância. A partir dessa estimativa pode-se determinar um alcance máximo para um enlace que não empregue compensação de PMD, considerando taxas de 10 e $40 \mathrm{~Gb} / \mathrm{s}$.
\end{abstract}

Palavras-Chave: Fibras Óticas, Dispersão do Modo de Polarização (PMD), Comunicação Ótica.

\begin{abstract}
We present a comparative study among statistical models for treatment of polarization mode dispcrsion in optieal links. With the obtained results it is possiblc to estimate the maximum allowed PMD coefficient to be found in long distance optical links. These values may be used to calculate the maximum possible distance a system may operate without PMD compensation considering 10 and $40 \mathrm{~Gb} / \mathrm{s}$ bit rates.
\end{abstract}

Keywords: Optical Fibers, Polarization Mode Dispersion, Optical Communication.

\section{INTRODUÇÃO}

A dispersão do modo de polarização (PMD Polarization Mode Dispersion) é um fator limitante para enlaces de comunicação ótica de longa-distância e operando a altas taxas de transmissão. Em uma fibra ótica cilindricamente perfeita existem dois modos de polarização que se propagam em direções perpendiculares com a mesma velocidade de grupo, conhecidos como estados de polarização principal. O afastamento da simetria cilíndrica provoca a qucbra dessa degenerescência, fazendo com que os dois modos se propaguem com velocidades de grupo diferentes ao longo dos eixos ortogonais, evidenciando um atraso teinporal, chamado de atraso diferencial de grupo (DGD - differential Group Delay). A quebra dessa simetria é oriunda de não-circularidades no núcleo da fibra geradas

C. E. P. Blume e A. A. P. Pohl estão com o Depto, de Eng. Elétrica (DAELN) e J. A. Medeitos Neto está com o Depto. de Eng. Mecânica (DAMEC) do Centro Federal de Educação Tecnológica do Paraná (CEFET-PR), Av. Sete de Setembro, 3165 CEP: 80.230-901 - Curitiba, Paraná. Tel: ++55 $413104695 /$ fax: ++55 41 31046 83. E-mails: ceblume@yahoo.com.br, pohl@cpgei.cefetpr.br, januncio@furukawa.com.br.

Editor de Área responsável: Antonio Sérgio Bezenra Sombra, Ricardo Menezes Campello de Souza e Max Gerken. Data de recebimento: $31 / \mathrm{Dez} / 2001$; data de revisão: $1 / \mathrm{Abr} / 2002$, data de aceitação: 5/Abr/2002. durante o processo de fabricação e devido à existência de stress não-simétrico resultante de microcurvaturas, torções, mudança de temperatura etc, aplicadas randomicamente ao longo da fibra ótica cabeada. Esscs mccanismos induzem o acoplamento de potência entre os dois modos de polarização principal, resultando em uma variação aleatória no atraso diferencial de grupo. O DGD é, portanto, uma função intrínseca do comprimento de onda e das condições ambientais as quais a fibra está submetida. Estatisticamente, o DGD segue uma distribuição de Maxwell [10]. Assim, uma medida única do DGD não permite caracterizar uma fibra. Ao invés disso, utiliza-se a figura da dispersão do modo de polarização (PMD) como uma mcdida da média ou da raiz quadrática média (ms) dos valores de DGD tomados sobre um intervalo de comprimentos de onda.

Existem vários estudos sobre o fenômeno de PMD. Contudo, apenas recentemente trabalhos sobre o PMD em fibras instaladas tem sido publicados [1,2,3]. Na prática, fibras são utilizadas dentro de cabos óticos. O processo de fabricação dos cabos e sua posterior instalação provocam microcurvaturas e torções adicionais na fibra alterando os valores de PMD. Em particular, a dificuldade em se mcdir o PMD em enlaces instalados tem suscitado a utilização de mćtodos estatísticos para se cstimar tal parâmetro $[4,5]$. Esses métodos empregam a abordagem de seções concatenadas de cabos para se calcular o PMD de um enlace de longa distância.

Neste trabalho apresentamos uma metodologia para a caracterização de enlaces óticos em relação ao parâmetro de PMD. Realizamos também uma análise comparativa de resultados obtidos a partir de medidas em cabos óticos com a configuração de tubo solto (loose tube), aplicando-se os modelos estatísticos descritos no boletim TIA/EIA TSB107 [6]. Os resultados numéricos demonstram a equivalência dos coeficientes de PMD máximo obtidos em função das técnicas aplicadas.

\section{COEFICIENTE DE PMD DE CABOS ÓTICOS}

O atraso diferencial de grupo (DGD) é um parâmetro geralmente medido na escala de picosegundos e representa o resultado singular de um proccsso estatístico. Por outro lado, o PMD representa o valor (médio) espcrado dessc mesmo processo. De forma prática, define-se um parâmetro denominado coeficiente de PMD como o valor de PMD dividido pela raiz quadrada do comprimento ( $\mathrm{ps} / \sqrt{ } \mathrm{km}$ ) de um lance de fibra ou cabo. Essa definição é consistente com resultados observados experimentalmente, onde $o$ valor de PMD assume, para longos comprimentos de fibra, uma dependência com a raiz quadrada da distância [12]. 
Os enlaces óticos são compostos por fibras cabeadas, garantindo, assim, maior segurança e durabilidade durante o tempo de vida útil do sistema. Porém, fibras cabeadas podem apresentar um valor de $\mathrm{PMD}$ diferente quando comparadas a fibras não cabeadas. Essa diferença é devida a existência de uma tensão de curvatura (bending strain) associada com parâmetros de projeto e fabricação do cabo ótico, tais como: o comprimento de passo, o diâmetro do membro central e o número de fibras.

Fabricantes de cabos são atualmente obrigados a caracterizar seus produtos quanto à dispersão do modo de polarização. Existem diversas técnicas que permitem acessar os parâmetros de DGD e PMD em uma fibra ou cabo ótico. Entre essas podeın ser citados o método da matriz de Jones, o método de varredura em comprimento de onda e o método interferométrico [7].

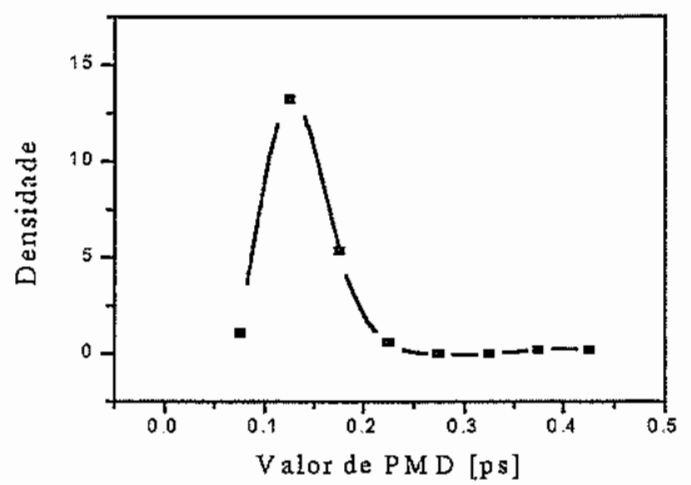

Figura 1. Distribuição dos valores de PMD medidos em uma seção de cabo de 24 fibras através da técnica de varredura em comprimento de onda.

A Fig. 1 mostra a distribuição dos atrasos (DGD) obtidos utilizando-se a técnica de varredura em comprimento de onda em um lance de cabo de 24 fibras com $5 \mathrm{~km}$ de comprimento na configuração de tubo solto. Nota-se que a distribuição segue claramente uma estatística maxwelliana, como previsto na teoria $[9,11]$.

\section{COEFICIENTE DE PMD DE ENLACES ÓTICOS}

A caracterização de um lance de cabo quanto ao PMD é insuficiente sob o ponto de vista prático. Um enlace de comunicação ótica é freqüentemente constituído por um grande número de lances de cabos. Cabos são fabricados com comprimentos de apenas alguns quilômetros e são emendados para formarem enlaces de longa distância. Fatores como o processo de instalação e as condições ambientais às quais os cabos do enlace são submetidos influenciam o valor de PMD. Devido ao seu caráter estatístico é necessário determinar um valor máximo de ocorrência de forma a garantir a performance do sistema de comunicação ótica.

$O$ coeficiente de PMD de um enlace pode ser definido como a raiz quadrada da soma dos quadrados dos coeficientes de PMD de cada seção de cabo dividido pelo número de seções que constituem o enlace.

$$
X_{N}=\sqrt{\frac{1}{N} \sum_{i=1}^{N} x_{i}^{2}}
$$

onde

$X_{N}$ - coeficiente de PMD do enlace (ps $/ \sqrt{\mathrm{km}}$ )

$x_{i}$ - coeficiente de PMD de uma seção de cabo $(\mathrm{ps} / \sqrt{\mathrm{km}})$

$N$-número de seções de cabo de um enlace

Como o coeficiente de PMD de cada seção de cabo é uma variável aleatória, o coeficiente de PMD do enlace também será.

Métodos estatísticos são empregados para tratar fenômenos de natureza estocástica, como no caso da dispersão do modo de polarização. Sua utilização implica na existência de um grande número de amostras, a partir das quais uma função de distribuição de probabilidade possa ser calculada. O trabalho, portanto, consiste em se obter esse número de amostras partindo-se dos valores experimentais obtidos em uma seção de cabo.

Como base amostral do processo estatístico foram utilizadas as medidas dos coeficientes de PMD obtidas em bobinas de $5 \mathrm{~km}$ de cabo óptico com 24, 72, 96 e 144 fibras na configuração de tubo solto. Foram realizadas medidas em 100 fibras escolhidas aleatoriamente, para cada configuração de cabo, empregando a técnica de varredura em comprimento de onda (wavelength-scanning) [7]. A tabela 1 mostra os valores médio, máximo e mínimo calculados a partir dos dados experimentais de um cabo de 24 fibras.

\begin{tabular}{|l|c|}
\hline & Coeficiente de PMD - Cabo de 24 Fibras \\
\hline Média & 0,065 \\
\hline Máximo & 0,196 \\
\hline Mínimo & 0,037 \\
\hline
\end{tabular}

Tabela 1. Valores experimentais para o coeficiente de $\mathrm{PMD}(\mathrm{ps} / \sqrt{\mathrm{km}})$

\section{MODEL.OS ESTATÍSTICOS}

Em uma distribuição estatística pode-se calcular o valor assumido por uma variável aleatória através da probabilidade de ocorrência daquele valor. Assim, a cada valor do coeficiente de PMD de uma dada distribuição, referente a um enlace com $N$ seções de cabos, está associada uma probabilidade de oconência.

Como consequîencia desse modelo estatístico pode-se, a partir da função densidade de probabilidade do enlace, derivar uma função confiabilidade. No caso específico de um enlace de comunicação ótica, cuja performance é degradada pela dispersão do modo de polarização, essa função confiabilidade deverá fornecer um valor de PMD, com uma baixa probabilidade de ocorrência, a partir do qual o sistema (por exemplo, taxa de bits) não será degradado.

Em particular, o coeficiente de PMD máximo do enlace, $X_{Q}$, pode ser definido em termos de um pequeno valor de probabilidade, $Q$, de tal forma que, para um dado valor de $Q$, a probabilidade de se encontrar um valor de coeficiente de PMD do enlace, $X_{N}$, maior do que $X_{Q}$ é menor do que a probabilidade $Q$ estipulada: 


$$
\operatorname{Pr}\left(X_{N}>X_{Q}\right)<Q
$$

$Q$ deve necessariamente assumir um valor baixo. No cálculos efetuados neste trabalho assumiu-se $Q<10^{-4}$.

A seguir descreve-se a construção da função densidade de probabilidade de um enlace constituído por $\mathrm{N}$ seções de cabos, onde foram utilizadas basicamente a técnica de Monte Carlo e a distribuição da função Gama.

\subsection{MÉTODO DE MONTE CARLO}

$\mathrm{Na}$ técnica de Monte Carlo, os coeficientes de PMD de cada seção de cabo, $x_{i}$, são escolhidos randomicamente, a partir dos valores experimentais. Esses valores são então empregados na equação (1) para se calcular o coeficiente de PMD de um enlace, $X_{N}$, composto por $N$ seções de cabo. Esse processo é repetido k vezes, onde $k \geq 10 / Q$. Como $Q$ deve ser um valor pequeno $\left(Q<10^{-4}\right)$, o processo é repetido no mínimo 100.000 vezes.

As Fig. 2 e 3 mostram a função densidade de probabilidade calculada através da técnica de Monte Carlo para enlaces de cabos de diversas configurações de fibras e constituídos por 10 e 200 seções de cabos, respectivamente. As figuras mostram a influência do número de seções no comportamento da distribuição. Verifica-se que o aumento do número de seções provoca uma redução na dispersão de cada distribuiçãa influenciando no cálculo do coeficiente máximo de $\mathrm{PMD}$, como será visto a seguir.

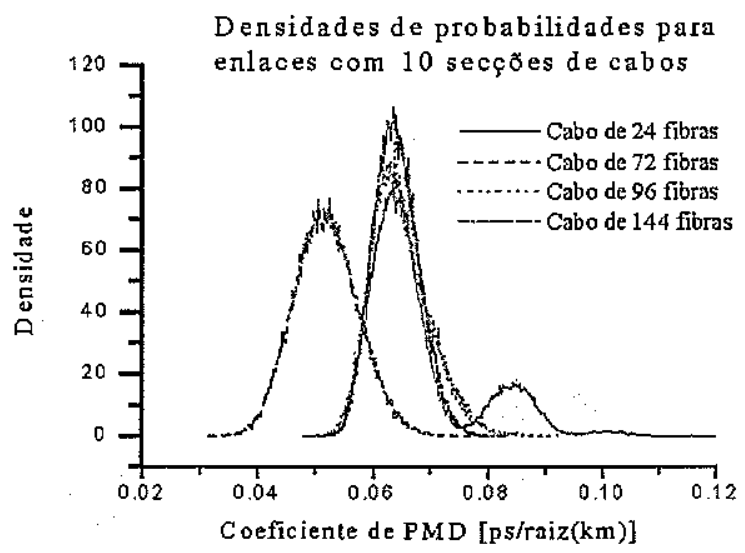

Figura 2. Distribuição dos coeficientes de PMD calculada pelo método de Monte Carlo para cabos com 24, 72, 96 e 144 fibras em um enlace com 10 seções $(50 \mathrm{~km})$.

O coeficiente de PMD máximo é determinado a partir da função de probabilidade acumulada

$$
c_{m}=\sum_{k=1}^{m} p_{k}
$$

onde $p_{k}$ representa a freqüência relativa normalizada na qual os valores do coeficiente de PMD do enlace aparecem na distribuição. O coeficiente de $\mathrm{PMD}$ máximo do enlace, $X_{Q}$, é encontrado pelo primeiro valor de $X_{N}$ que satisfaça a condição $c_{m} \geq 1-Q$.
Através desses cálculos os valores de PMD admissiveis são aqueles que se encontram no final da curva de distribuição de densidade de probabilidade. Se a curva sofie

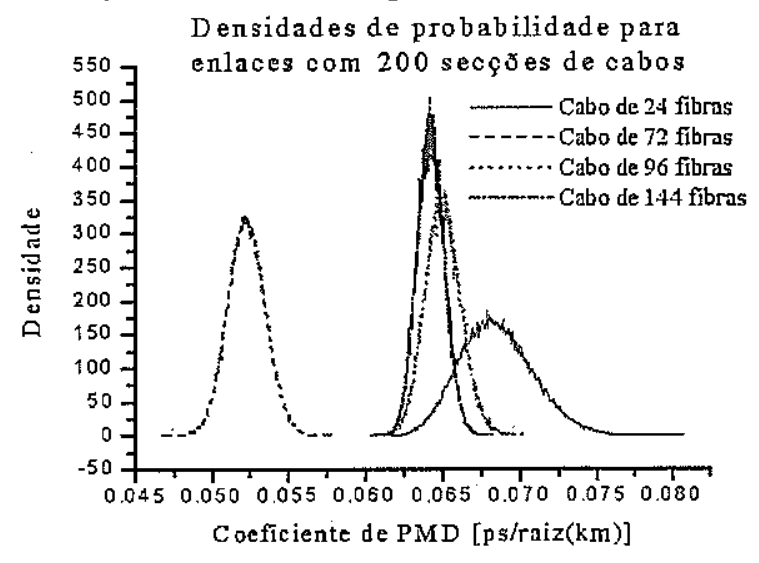

Figura 3. Distribuç̧ão dos coeficientes de PMD calculada pelo método de Monte Carlo para cabos com 24, 72, 96 e 144 fibras em um enlace com 200 seções (1000 km).

uma compressão devido ao aumento no número de seções, os valores de PMD admissíveis também serão alterados. $\mathrm{Na}$ prática, $o$ fato de se ter um enlace longo significa estatisticamente que os valores máximos de $\mathrm{PMD}$ observados serão menores do que os de um enlace bem mais curto. Como exemplo, tome-se o cabo de 72 fibras, onde, para 10 seções (correspondente a $50 \mathrm{~km}$ ), obtém-se valores de $\mathrm{PMD}$ a partir de $0.060 \mathrm{ps} / \sqrt{\mathrm{km}}$ enquanto para o enlace com 200 seções $(1000 \mathrm{~km})$ obtém-se valores a partir de $0.055 \mathrm{ps} / \sqrt{\mathrm{km}}$.

\subsection{FUNÇÃO GAMA}

A segunda técnica utilizada é a da função Gama $[13,14,15]$, que é um modelo utilizado para representar vários fenômenos físicos, inclusive a distribuição dos coeficientes de PMD em um enlace ótico. O modelo baseiase no pressuposto de que o quadrado do coeficiente de PMD das seções individuais de cabos óticos segue uma distribuição de acordo com uma variável randômica do tipo Gama. Assim, a distribuição dos coefícientes de PMD de um enlace com várias seções de cabos concatenados também poderá ser representado através da mesma variável. A partir desse fato, pode-se estimar os parâmetros que definem a distribuição de probabihidades para o enlace.

A função de distribuição de probabilidades Gama, ou função densidade de probabilidade, é expressa por:

$$
f(X, \alpha, \beta, N)=2 \frac{(N \beta)^{M \alpha}}{\Gamma(\alpha)} X^{2 M \alpha-1} \exp \left[-N \beta X^{2}\right]
$$

onde, $X$ é o coeficiente de PMD do enlace, $\alpha$ e $\beta$ são parâmetros que definem, respectivamente, a forma e a escala da distribuição e $N$ é o número de seções de cabos que se deseja concatenar para formar o enlace.

Para a caracterização da distribuição Gama, necessita-se atribuir valores numéricos aos parâmetros $\alpha$ e $\beta$. Isto pode 
ser feito utilizando-se o Método dos Momentos [6] ou o Método da Máxima Verossimilhança $[4,16]$, descritos a seguir.

\subsubsection{MÉTODO DOS MOMENTOS}

Este método é baseado na premissa de que $N \alpha \geq 5$ e também é considerado interativo, pois os parâmetros são estimados antes que a verificação da desigualdade possa ser feita. Para realizar essa estimativa necessita-se empregar novamente a técnica de Monte Carlo com o objetivo de assegurar que a desigualdade acima seja satisfeita. Da teoria estatística [13] tem-se que o valor esperado ou a média da distribuição Gama (momento de $1^{\text {a }}$ ordem em relação à origem da distribuição) é dada por $\alpha / \beta$. A técnica de Monte Carlo permite gerar vários valores para o coeficiente de PMD do enlace, calcular a média dos quadrados e comparar com a média da função Gama, como descrito na Equação 4:

$$
\frac{\alpha}{\beta}=v^{2}=\frac{1}{k} \sum_{i=1}^{k} X_{i}^{2}
$$

onde, $k$ é o número de interações de Monte Carlo ( > 100.000)

A partir do cálculo da variância da função Gama, podese aproximá-la pela variância em relação a média dos quadrados (momento de $2^{\text {a }}$ ordem em relação à média da distribuição) calculada pelos pontos de Monte Carlo, ou seja:

$$
\frac{1}{4 N \beta} \cong E\left[(X-v)^{2}\right]
$$

Assim,

$$
\frac{1}{4 N \beta}=\frac{1}{k} \sum_{i=1}^{k}\left[X_{i}-v\right]^{2}
$$

Com as Equações (4) e (6) os parâmetros $\alpha$ e $\beta$ estarão estimados. Finalmente, a distribuição dos coeficientes de PMD poderá ser construída utilizando-se a equação 3 .

\subsubsection{MÉTODO DA MÁXIMA VEROSSIMILHANÇA}

Esse método parte diretamente dos coeficientes de PMD medidos nas seções individuais de cabos. Define-se uma função log-verossimilhança [16] dada por:

$$
\begin{aligned}
& G\left(\alpha, \beta, x_{i}\right)=\sum_{i=1}^{n} \ln \left(\frac{2}{x_{i}}\right)-n \ln (\Gamma(\alpha)) \div \\
& +\alpha \sum_{i=1}^{n} \ln \left(\beta x_{i}^{2}\right)-\beta \sum_{i=1}^{n} x_{i}^{2}
\end{aligned}
$$

onde $\underline{n}$ é o número de medidas realizadas nas seções individuais ( 100 medidas, no caso deste trabalho) e $x_{i}$ é o coeficiente de PMD de uma secção de cabo.

Os valores estimados de $\alpha$ e $\beta$ são aqueles que maximizam a função $G$. Para isto, deriva-se $G$ parcialmente em relação a $\alpha$ e $\beta$ e iguala-se a zero. Simplificando as equações, obtém-se:

$$
\ln (\alpha)-\psi(S n)+2 g n=0
$$

$$
\beta=\alpha / S n
$$

onde,

$$
S n=\frac{1}{n} \sum_{i=1}^{n} x_{i}^{2}, \quad g n=\frac{1}{n} \sum_{i=1}^{n} \ln \left(x_{i}\right)
$$

e $\psi(\alpha)$ é a função DiGama $[14,15]$. A equação 8 é resolvida calculando-se a raiz da função para $\alpha$. Finalmente, a distribuição dos coeficientes de PMD poderá ser construída utilizando-se a equação 3.

\subsubsection{CÁLCULO DO PMD MÁXIMO DO ENLACE}

Para um dado valor de $Q$, a probabilidade de se encontrar um valor de coeficiente de PMD maior do que $X_{Q}$ é calculada através da integral

$$
\int_{0}^{p_{Q}} \frac{y^{a-1}}{\Gamma(a)} \exp (-y) d y=1-Q
$$

onde, $a \equiv N \alpha$ e $y \equiv N \beta\left(X_{N}\right)^{2}$. N representa o número de seções de cabos e $X_{N}$ é o coeficiente de PMD do enlace. $\alpha$ e $\beta$ são os parâmetros de formato e escalonamento determinados através dos métodos acima.

$\mathrm{Na}$ solução dessa integral, deve-se encontrar um valor para $p_{Q}$ de tal forma que a condição $1-Q$ seja atendida.

$\mathrm{O}$ coeficiente de $\mathrm{PMD}$ máximo do enlace pode ser então calculado pela expressão:

$$
X_{\underline{Q}}=\left(\frac{p_{Q}}{N \beta}\right)^{1 / 2}
$$

\subsection{TEOREMA DO LIMITE CENTRAL}

A técnica do Teorema do Limite Central Generalizado parte diretamente dos coeficientes de PMD medidos em seções individuais de cabos, a partir dos quais os seguintes momentos são calculados

$$
\begin{gathered}
\mu_{\mathrm{I}}=\frac{1}{n} \sum_{i=1}^{n} x_{i}^{2} \\
\mu_{2}=\frac{1}{n-1} \sum_{i=1}^{n}\left(x_{i}^{2}-\mu_{1}\right)^{2} \\
\mu_{3}=\frac{1}{n-1} \sum_{i=1}^{n}\left(x_{i}^{2}-\mu_{1}\right)^{3}
\end{gathered}
$$

onde 
$x_{i}$ - coeficiente de PMD medido em uma seção de cabo $(\mathrm{ps} / \mathrm{kmm})$

$\underline{n}$ - número de medidas realizadas

O coeficiente de PMD máximo do enlace, partindo-se da função densidade de probabilidade acumulada [8], pode ser então calculado como

$$
x_{Q} \approx\left[\mu_{1}+z_{Q}\left(\frac{\mu_{2}}{N}\right)^{1 / 2}+\frac{\mu_{3}}{6 \mu_{2} N}\left(z_{Q}^{2}-1\right)\right]^{1 / 2}
$$

onde $z_{Q}$ é uma variável que depende do valor atribuído a $Q$ [6]. Na equação (15), $N$ indica o número de seç̃̃es de cabos utilizado.

\section{RESULTADOS}

O presente estudo visa sobretudo a aplicação dos métodos descritos acima coln o objetivo de se verificar sua compatibilidade, coerência de resultados e facilidade de aplicação.

A Fig. 4 mostra a distribuição dos coeficientes de PMD calculada pelos métodos de Monte Carlo e Função Gama para um enlace composto de 10 seções de um cabo com 24 fibras. Verifica-se uma grande discrepância nas distribuições, evidenciada pelo deslocamento entre as freqüências máximas.

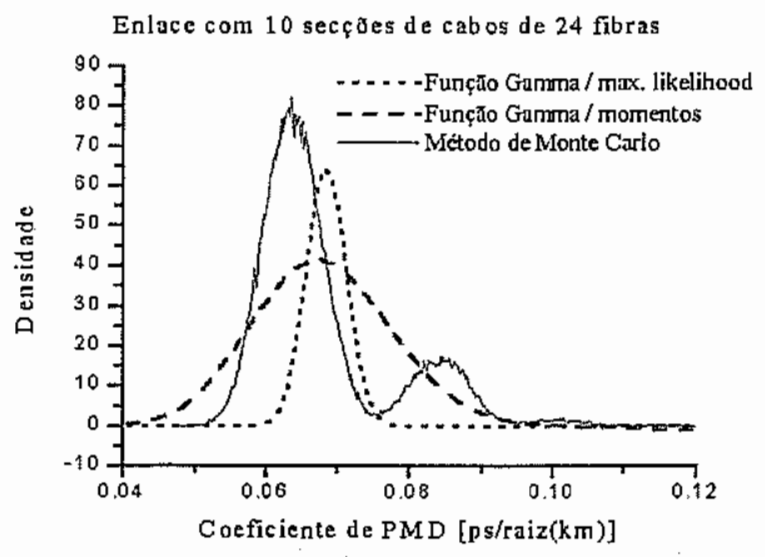

Figura 4. Distribuições dos coeficientes de PMD através de Monte Carlo e Função Gama para um cabo de 24 fibras em um enlace com 10 seções $(50 \mathrm{~km})$.

A Fig. 5 mostra esse mesmo cálculo considerando, porém, um enlace constituído por 50 seções. Nota-se neste caso que as distribuições começam a se concentrar sobre um mesmo valor central de coeficiente de $\mathrm{PMD}$.

Aumentado-se o número de seções para 200 (Fig. 6) observa-se que os modelos de Monte Carlo e da função Gama, através do método dos momentos, apresentam uma distribuição praticamente idêntica, evidenciando um resultado fundamental da estatística dado pelo seu Teorema do Limite Central. Esse teorema afirnna que distribuições distintas tendem a uma distribuição do tipo normal (gaussiana) quando o número de pontos da distribuição tende a infinito [13]. Contudo, o modelo da função Gama, considerando a técnica da máxima verossimilhança, não conduz ao mesmo resultado, embora o valor máximo de coeficiente de PMD praticamente coincida com o calculado pelos outros dois modelos.

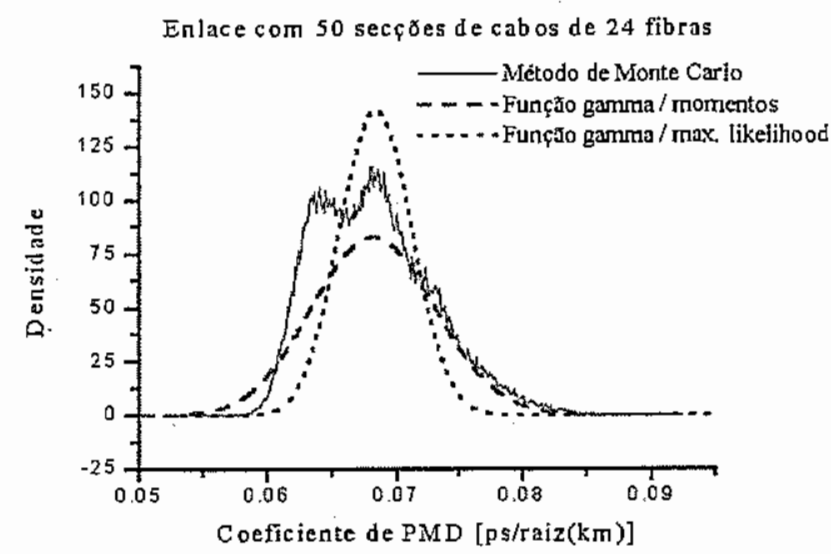

Figura 5. Distribuições dos coeficientes de PMD através de Monte Carlo e Função Gama para um cabo de 24 fibras em um enlace com 50 seções $(250 \mathrm{~km})$.

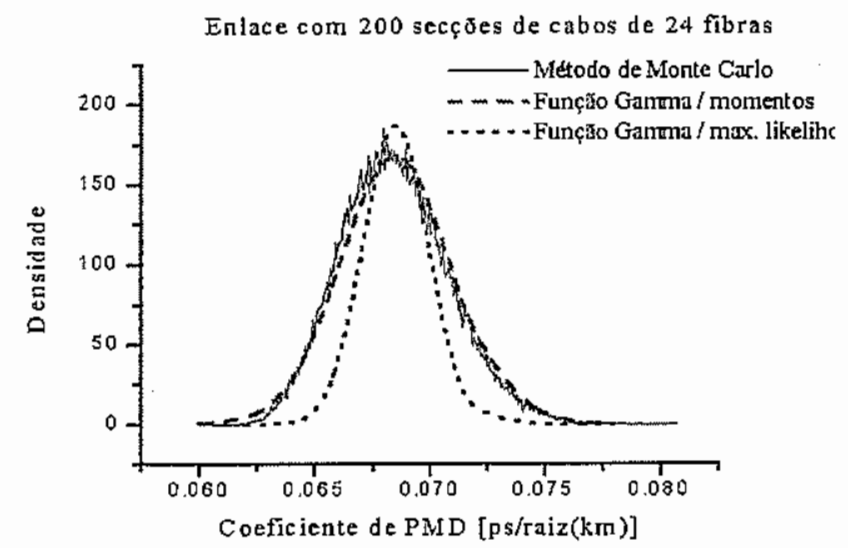

Figura 6. Distribuições dos coeficientes de PMD através de Monte Carlo e Função Gama para uun cabo de 24 fibras em um enlace com 200 seções $(1000 \mathrm{~km})$.

Por outro lado, considerando o cabo com 72 fibras e aplicando os métodos descritos, é interessante observar uma convergência entre os resultados mesmo para um enlace composto por apenas 10 seções de cabo, como pode ser visto na Fig. 7.

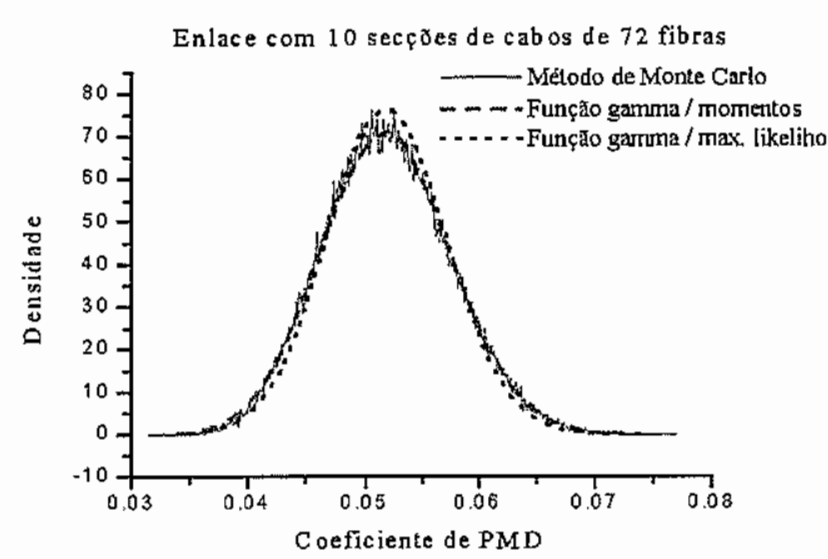

Figura 7. Distribuições dos coeficientes de PMD através de Monte Carlo e Função Gama para um cabo de 72 fibras em um enlace com 10 seçôes $(50 \mathrm{~km})$. 
A Fig. 8 mostra a distribuição dos coeficientes de PMD para um enlace composto por 200 seções do mesmo cabo. Nota-se novamente que a dispersão dos coeficientes se reduz com o aumento do número de seções. Na prática, esse resultado pode ser associado a uma freqüência maior de acoplamento de potência ótica entre os modos de propagação ao longo do enlace, induzindo uma equalização dos atrasos observados.

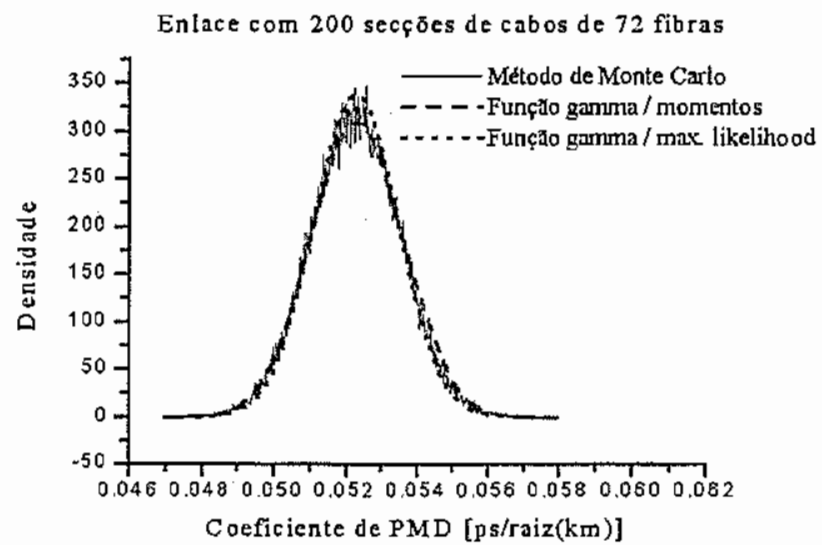

Figura 8. Distribuições dos coeficientes de PMD através de Monte Carlo e Função Gama para um cabo de 72 fibras em um enlace com 200 seções $(1000 \mathrm{~km})$.

A Fig. 9 mostra o comportamento do coeficiente de PMD máximo, calculado pelas três técnicas, em função do número $N$ de seções de cabos utilizados para calcular o coeficiente de PMD do enlace, $X_{Q}$. As curvas foram calculadas empregando-se um valor de $10^{-4}$ para 0 parâmetro $Q$. Cada seção de cabo representa um comprimento de $5 \mathrm{~km}$. Da figura, observa-se a convergência do coeficiente de PMD máximo admissivel, $X_{Q}$, para valores de $N>20$. A Tabela 2 mostra os valores dos coeficientes de PMD máximos calculados para um enlace com $N=20$

\begin{tabular}{|l|c|}
\hline & Coeficiente de PMD Máximo $-X_{Q}$ \\
\hline Monte Carlo & 0,105294 \\
\hline Função Gama & 0,106144 \\
\hline $\begin{array}{l}\text { Teorema do } \\
\text { Limite Central }\end{array}$ & 0,106415 \\
\hline
\end{tabular}

Tabela 2. Valores do coeficiente de PMD máximo para um enlace composto por 20 seções de cabos de $5 \mathrm{~km}$.

A fim de acessar o impacto do coeficiente máximo de PMD na performance de um sistema de comunicação ótica é necessário associar esse parâmetro com a máxima distância do enlace e a taxa de transmissão. Isto pode ser feito definindo-se um pequeno valor de probabilidade, a partir do qual o alargamento do pulso dentro do slot de bit, provocado pela dispersão do modo de polarização (coeficiente máximo de $\mathrm{PMD}$ admissível), não degradaria a performance do sistema. Essa probabilidade é expressa como [4]:

$$
P\left[X>\frac{f_{a} \cdot 1000}{B \cdot \sqrt{\text { Lmáx }}}\right]=10^{-4}
$$

onde

$$
L m a ́ x=\left(\frac{1000 \cdot f_{a}}{B \cdot X m a ́ x}\right)^{2}
$$

e $f_{a}$ representa a tolerância admitida para o alargamento temporal do pulso no slot de bit.

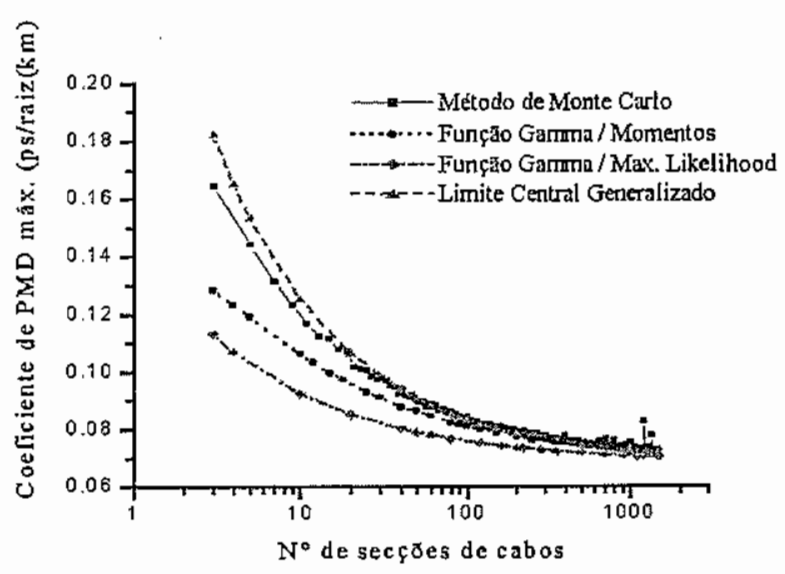

Figura 9. Coeficiente de PMD máximo $(\mathrm{ps} / \sqrt{\mathrm{km}})$ de um enlace em função do número $N$ de seções de cabos utilizadas nos modelos estatísticos.

A Fig. 10 mostra o comportamento do comprimento máximo do enlace em função do coeficiente de PMD máximo admissível, não levando em consideração a dispersão cromática na fibra (ou seja, a dispersão cromática deve ser nula ou previamente compensada). A simulação foi realizada com as taxas de 10 e $40 \mathrm{~Gb} / \mathrm{s}$ supondo-se uma tolerância $f_{a}$ entre 10 e $15 \%$ do alargamento temporal do pulso dentro do slot de bit. É interessante observar a forte dependência do coeficiente de PMD com a taxa de bits. $\mathrm{O}$ aumento da taxa de transmissão ( $>40 \mathrm{~Gb} / \mathrm{s}$ ) implica em uma redução dramática do comprimento do enlace, se o sistema não for compensado.

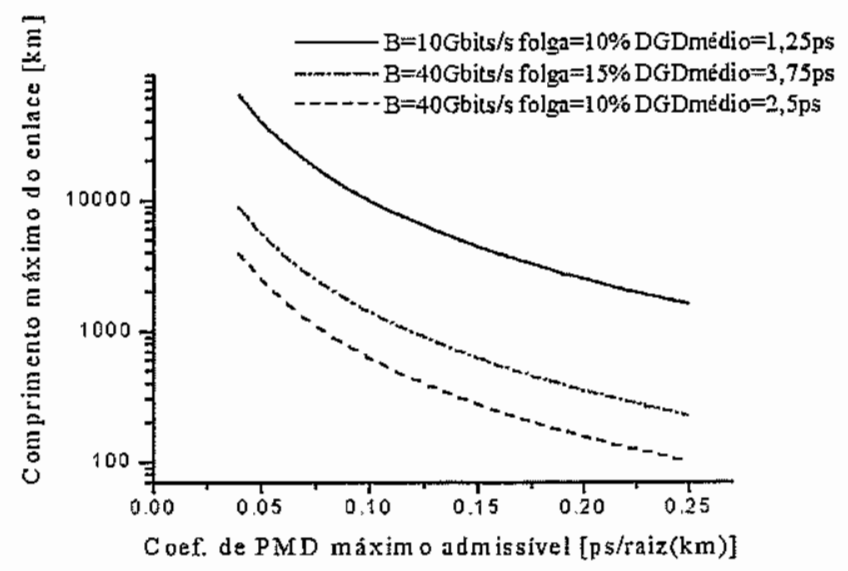

Figura 10. Alcance máximo de um enlace em função das taxas de transmissão. 


\section{CONCLUSÃO}

Através da utilização de diferentes modelos estatísticos foi possível construir funções de distribuição para o coeficiente de PMD de enlaces de óticos, a partir de dados obtidos experimentalmente em cabos com comprimento de $5 \mathrm{~km}$ na configuração tubo loose.

Os resultados mostraram que a partir de um determinado número de seções $(N>20)$ a variação no valor do coeficiente de PMD máximo converge para um determinado valor, qualquer que seja o modelo estatístico empregado. Contudo, resultados obtidos para $N<10$. mostraram uma razoável variação nos valores estimados.

Foi observado uma forte dependência do coeficiente de PMD com a taxa de bits. $O$ aumento da taxa de transmissão (> $40 \mathrm{~Gb} / \mathrm{s}$ ) implicou em uma redução significativa do comprimento do enlace, quando o sistema não for compensado. Essa redução mostra a necessidade, do ponto de vista prático, de compensação do fenômeno de PMD operando a altas taxas de transmissão.

\section{AGRADECIMENTOS}

À Furukawa Industrial S.A. pelo apoio financeiro através de projeto financiado sob os auspícios da Lei de Informática.

\section{REFERÊNCIAS}

[1] H. Sunnerud, B.-E. Olsson, M. Karlsson, P.A. Andrekson e J. Brentel,"Polarization-Mode Dispersion Measurements Along Installed Optical Fibers Using Gated Backscattered Light and a Polarimeter", J. Lightwave Technol., vol. 18, pp. 897-904, Julho 2000.

[2] M. Karlsson, J. Brentel e P.A. Andrekson, "Long-Term Measurement of PMD and Polarization Drift in Installed Fibers", J. Lightwave Technol,, vol. 18, pp. 941-951, Julho 2000.

[3] A. Galtarossa, L. Palmieri, A. Pizzinat, M. Schiano e T. Tambosso, "Measurement of Local Beat Length and Differential Group Delay in Installed Single-Mode Fibers", J. Lightwave Technol., vol. 18, pp. 1389-1394, Outubro 2000.

[4] S.A. Jacobs, J.J. Refi e R.E. Fangmann, "Statistical Estimation of PMD Coefficients for System Design", Electron. Lett., vol. 33, pp. 619-621, Março 1997.

[5] L.F. Marques, A. M. Simião, R. F. Cruz, J.A. Medeiros e M.R. Boulos,"Statistical Analyses of PMD Using Monte Carlo Method for Different Configuration of Loose Tube Optical Cable", in Proceedings of OFC'2001, 2001, paper WDD12

[6] TIA/EIA TSB107. "Guidelines for the Statiscal Specification of Polarization Mode Dispersion on Optical Fiber Cables", edição de Novembro 1999

[7] R. Cross, "PMD Measurements Methods", Fiberoptic Product News, pp.27-29, Junho 1995.
[8] J. Patel e C. Read, "Handbook of the Normal Distribution", Marcel Delkker Inc., New York, 1982.

[9] B. W. Hakki, "Polarization Mode Dispersion in a Single Mode Fiber", J. Lightwave Technol., vol. 14, no. 10, pp. 2202-2208, 1996.

[10] F. Curti, B. Daino, G. DeMaris and F. Matera, "Statiscal Treatment of the Evolution of the Principal States of Polarization in Single-Mode Fibers", J. Liglhtwave Technol., vol. 8, pp. 1162-1166, Agosto 1990.

[11] N. Gisin, R. Passy, J. C. Bishoff and B. Perny, "Experimental Investigations of the Statistical Properties of Polarization Mode Dispersion in Single Mode Fibers", IEEE Photonics Techn. Lett., vol. 5, no. 7, pp 819-821, 1993.

[12] N. Gisin, J.-P. Van der Weid and J.-P. Pellaux, "J. Lightwave Technol., vol. 9, no. 7, pp. 821-827, 1991.

[13] P. L. Meyer, "Probabilidade: aplicações à estatística", Livros Técnicos e Científicos Editora, Rio de Janeiro, 1981.

[14] Abramowitz, M.., e Stegun, I.: 'Handbook of Mathematical Functions', Dover Publications, New York, 1970.

[15] Press, W., Teukolsky, S., Vetterling, W., e Flanner Y, B.: 'Numerical Recipes in Fortran', Cambridge University Press, 1992.

[16] Breimann, L.: 'Statistics: with a view toward applications' Houghton Mifflin Company, Boston, 1973.

C. E. P. Blume é estudante de engenharia mecânica do CEFET$\mathrm{PR}$, desde 1999. No momento é aluno de iniciação científica do projeto de pesquisa do convênio Funcefet-PR - Furukawa Industrial S.A. Trabalha na área de pesquisa e desenvolvimento de propriedades mecânicas e óticas de fibras óticas. Também é formado no curso Técnico em Mecânica pelo CEFET-PR

A.A.P. PohI graduou-se em Física pela Universidade Estadual de Campinas. (1983) onde também obteve o título de Mestre em Física (1987). De 1987 a 1989 trabalhou como pesquisador assistente no Instituto de Estudos Avançados do Centro Técrico Aeroespacial èm São José dos Campos. De 1990 a 1994 trabalhou como pesquisador visitante no Instituto de Altas Freqüências da Universidade Técrica de Braunschweig, Alemanha, onde obteve o título de Doutor em Engenharia Elétrica. De 1995 a 2000 atuou na área de sistemas na empresa Furukawa Industrial S.A. Desde de Janeiro de 2001 é professor adjunto do departamento de eletrônica do CEFET-PR. Sua principal área de interesse está atualmente voltada para sistemas e redes de comunicação ótica.

J. A. Medeiros Neto graduou-se em Física pela Universidade do Rio Grande do Norte (UFRN). É mestre e doutor pela Universidade de Campinas (UNICAMP). Após concluir pósdoutorado na ORC - University of Southhampton - Inglaterra, ingressou na UNICAMP como pesquisador associado. Em 1998, ingressou no Centro de Educação Tecnológica do Paraná (CEFETPR) como pesquisador e na Furukawa Industrial S.A. como consultor. Trabalhou em várias áreas de Ciência dos Materiais Vítreos como non-oxide glass para fibras ópticas e para confiabilidade de componentes. 